\title{
WHO-INRUD Drug Use Indicators in Two Tertiary Healthcare Facilities in Midwestern Nigeria
}

\author{
Sunday O Oghuvwu ${ }^{1}$, Ambrose O Isah ${ }^{1,2}$ \\ ${ }^{I}$ Department of Internal Medicine, University of Benin Teaching Hospital, PATS MECOR, Nigeria, ${ }^{2}$ Department of \\ Clinical Pharmacology and Therapeutics, University of Benin, Benin City, Nigeria
}

\section{BACKGROUND}

Periodic assessment of prescribing patterns, patient care practices and facility indicators (using the WHO-INRUD drug use indicators) is essential in identifying the extent of rational use of medicines and standard of care in healthcare facilities. The study assessed these features in facilities in Midwestern Nigeria.

METHODS

A cross-sectional descriptive study of 1800 prescriptions from January to December 2014 and 600 patient encounters from two tertiary hospitals were evaluated using the WHO drug use indicator tool. Data on prescribed medicines during the clinical encounters; consultation and dispensing times during the respective encounters; proportion of dispensed and appropriately labelled drugs; as well as information obtained during an interview for knowledge of dosage were all collected. Data on availability of copies of EML and key drugs in both facilities were also obtained. Information obtained were recorded in a spreadsheet and then analyzed according to WHO guidelines.

RESULTS

The average number of medicines per encounter were 2.8+_1.6. The percentage of generic prescription was $49.6 \%$ while percentage of encounters with antibiotics, antimalarials and injections were $27.4 \%, 23.2 \%$ and $12.4 \%$ respectively. Prescription of medicines listed in the EML was $66.6 \%$. Average consulting and dispensing times were 15.3 minutes and 136 seconds respectively. Percentage of medicines actually dispensed was $86.2 \%$ while $98.7 \%$ of medicines were adequately labelled. Knowledge of correct dosage of prescribed medicines was $96.5 \%$. Availability of key medicines and at least a copy of EML was $80 \%$ and $0 \%$ respectively.

\section{CONCLUSION}

Gaps still exist in the level of rational use of medicines in the area. The result is a reflection of the situation across the country and calls for sustained campaign to optimize rational use of medicines and the level of patient care practices. 